\section{Diät bei}

\section{Herzinsuffizienz?}

Für die Herzinsuffizienz mit erhaltener Auswurffraktion (HFPEF) gibt es derzeit keine evidenzbasierte medikamentöse Therapie. Die Behandlung beschränkt sich auf die Kontrolle von Risikofaktoren und die Linderung der Beschwerden. Nach neuen Erkenntnissen von US-Ärzten könnten die meist übergewichtigen Patienten auch selbst dazu beitragen, das Hauptsymptom - die Belastungsintoleranz - zu reduzieren. In einer Studie mit adipösen HFPEF-Patienten erwiesen sich sowohl eine kalorienreduzierte Ernährung als auch ein Ausdauertraining als wirksam. Bei einer Kombination der beiden Maßnahmen addierten sich die Effekte sogar.

\section{Compliance: Fragen ist Gold}

Die Kardiologen Rosemary Hines und Neil Stone, USA, haben 21 Kollegen und $66 \mathrm{~Pa}$ tienten über ihre Gewohnheiten in puncto kardioprotektiver, medikamentöser Behandlung befragt. 40 Patienten (61\%) berichteten, selten oder nie mit ihren Ärzten darüber zu sprechen, ob sie die verordneten Mittel auch einnehmen. 18 von diesen (45\%) räumten ein, die Tabletteneinnahme manchmal oder üblicherweise zu vergessen. Doch die Prüfung der Therapietreue koste nur wenige Sekunden. Die Kardiologen empfehlen, Patienten die folgende Frage zu stellen: „Wie oft haben Sie in den vergangenen 30 Tagen vergessen, ihre Herzmedikamente einzunehmen?"

Hines R, Stone NJ et al. JAMA Cardiol 2016 (online first)

\section{Antikoagulation per Ampelsystem}

Flimmert der Vorhof, droht ein Schlaganfall - vor allem bei älteren Patienten ist VHF eine häufige Ursache für Insulte. Um es gar nicht erst soweit kommen zu lassen, stehen verschiedene Antikoagulanzien als Präventionsmaßnahme zur Verfügung. Doch wann und für wen sind die Medikamente tatsächlich indiziert? Erste Anhaltspunkte für die Entscheidung bietet eine "Ampeltabelle" - was hier rot, gelb und grün bedeuten, lesen Sie in unserem Schwerpunkt „Kardiologie“.

Claudia Daniels

Redakteurin

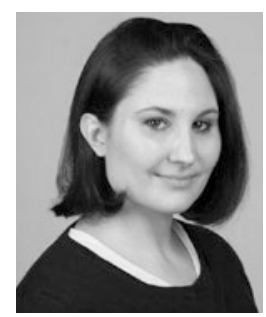

\title{
Elektrodenloser Mini-Schrittmacher reduziert Komplikationsrate
}

\section{Migräne: Herz und Hirn sind in Gefahr}

Sie sind so groß wie eine Füllerpatrone und damit um mehr als $90 \%$ kleiner als herkömmliche Schrittmachersysteme, sie kommen ohne transvenöse Elektroden aus und lassen sich minimalinvasiv implantieren - miniaturisierte Herzschrittmacher könnten, so die Hoffnung, Komplikationen, wie sie unter herkömmlichen Schrittmachersystemen beobachtet werden, hinfällig machen. Denn diese Miniaggregate werden via Katheter komplett in den rechten Vent- rikel eingeführt und an der Wand des rechten Ventrikels verankert, wodurch die Infektionsgefahr sinkt und Elektrodendisklokationen entfallen. Beispielsweise wurden bei 726 Teilnehmern einer internationalen Kohortenstudie nach 7,7 Monaten der Implantation des Micra Transkatheter-Schrittmachersystems keinerlei Sondendislokationen und Infektionen registriert.

Late-Breaking Devices, Cardiostim 2016, Nizza

\section{Blutdruck-Auf-und-Ab geht auf den Geist}

In den letzten Jahren hat man erkannt, dass die Blutdruckvariabilität prognostisch relevant ist. Größere Differenzen zwischen monatlichen oder jährlichen Praxismessungen sind beispielsweise mit einem erhöhten Risiko für Schlaganfälle assoziiert. Gleichzeitig scheinen sie mit einem beschleunigten geistigen Abbau einherzugehen. Dieser Zusammenhang wurde in einer prospektiven Studie bei älteren Menschen gefunden. Die Studienkohorte bestand aus einer Zufallsstichprobe von 976 über 54-Jährigen, bei denen bei mindestens drei Untersuchungen im Abstand von drei Jahren der Blutdruck bestimmt und danach bei mindestens zwei weiteren Terminen die kognitive Leistungsfähigkeit untersucht worden war. Letztere wurde anhand eines standardisierten Tests beurteilt. Verglichen mit Patienten aus dem Terzil mit der geringsten Variabilität des systolischen Blutdrucks verloren Patienten aus dem mittleren und oberen Terzil im Lauf von fünf Jahren 0,13 bzw. 0,26 Punkte mehr im globalen kognitiven Score.

Qin B et al. Hypertension 2016, online 23. Mai
Der Zusammenhang zwischen Migräne und Schlaganfällen ist schon lange etabliert. Inzwischen mehren sich die Belege, dass sich das erhöhte Risiko auch auf andere kardiovaskuläre Komplikationen erstreckt. Laut einer Auswertung der Nurses Health Study II haben junge Frauen, die an Migräne leiden, längerfristig ein um 50\% höheres Risiko für schwere kardiovaskuläre Ereignisse als Frauen ohne Migräne. Die Studienautoren um Prof. Dr. Tobias Kurth, Berlin, fordern deswegen dazu auf, Migränepatientinnen auch im Hinblick auf ihr vaskuläres Risiko zu untersuchen. Die 115.541 Teilnehmerinnen der Nurses Health Study II waren zwischen 25 und 42 Jahre alt und frei von Herz-Kreislauf-Erkrankungen. 17.531 hatten zu Beginn bereits eine ärztlich diagnostizierte Migräne, weitere 6389 kamen im Lauf der Studie hinzu. Während der über 20-jährigen Nachbeobachtungszeit wurden 678 Herzinfarkte, 651 Schlaganfälle und 223 Todesfälle aus kardiovaskulärer Ursache erfasst. Nach Bereinigung einiger Risikofaktoren hatten Migränepatientinnen ein um $50 \%$ erhöhtes Risiko für ein schweres kardiovaskuläres Ereignis.

Kurth Tet al. BMJ 2016;353:i2610

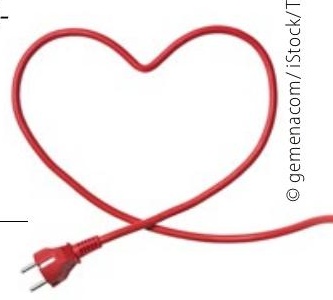

